\title{
Model-Free Plug-n-Play Optimization Techniques to Design Autonomous and Resilient Complex Systems
}

\author{
K. G. Vamvoudakis ${ }^{1}$, Member, IEEE J. P. Hespanha ${ }^{1}$, Fellow, IEEE
}

\begin{abstract}
This talk will focus on model-free distributed optimization based algorithms for complex systems with formal optimality and robustness guarantees. Given the presence of modeling uncertainties, the unavailability of the model, the possibility of cooperative/non-cooperative goals and malicious attacks compromising the security of networked teams, there is a need for completely model-free plug-n-play approaches that respond to situations not programmed or anticipated in design, in order to guarantee mission completion. Unfortunately, existing schemes for complex systems do not take into account recent advances of computational intelligence. This talk will combine interdisciplinary ideas from different fields, i.e. computational intelligence, game theory, control theory, and information theory to develop new self-configuring algorithms for decision and control given the unavailability of model, the presence of enemy components and the possibility of measurement and jamming network attacks. Due to the adaptive nature of the algorithms, the complex systems will be capable of breaking or splitting into parts that are themselves autonomous and resilient. The proposed algorithms will be provided with guaranteed optimality and robustness and will be able to enable complete on-board autonomy, to multiply engagement capability, and enable coordination of distributed, heterogeneous teams of manned/unmanned vehicles and humans.
\end{abstract}

\section{REFERENCES}

[1] K. G. Vamvoudakis, J. P. Hespanha, B. Sinopoli, Y. Mo, "Detection in Adversarial Environments," IEEE Transactions on Automatic Control (Special Issue on Control of Cyber-Physical Systems), vol. 59, no. 12, pp. 3209-3223, 2014

[2] K. G. Vamvoudakis, J. P. Hespanha, R. A. Kemmerer, G. Vigna, "Formulating Cyber-Security as Convex Optimization Problems," in Control of Cyber-Physical Systems, Lecture Notes in Control and Information Sciences, ed. Danielle Tarraf, Volume 449, pp. 85-100, Springer-Verlag, Berlin, 2013

[3] K. G. Vamvoudakis, F. L. Lewis, G. R. Hudas, "Multi-Agent Differential Graphical Games: Online Adaptive Learning Solution for Synchronization with Optimality," Automatica, vol. 48, no. 8, pp. 15981611,2012

[4] K. G. Vamvoudakis, F. L. Lewis, "Online Actor-Critic Algorithm to Solve the Continuous-Time Infinite Horizon Optimal Control Problem," Automatica, vol. 46, no. 5, pp. 878-888, 2010

[5] D. Vrabie, K. G. Vamvoudakis, F. L. Lewis, Optimal Adaptive Control and Differential Games by Reinforcement Learning Principles, Control Engineering Series, IET Press, 2012

\footnotetext{
*This material is based upon work supported in part by ARO MURI Grant number W911NF0910553.

${ }^{1}$ K. G. Vamvoudakis, and J. P. Hespanha are with the Center for Control, Dynamical-systems and Computation (CCDC), University of California, Santa Barbara, CA 93106-9560 USA e-mail: kyriakos@ece.ucsb.edu.
} 